\section{Tracheal stenosis and recurrent nerve paralysis due to thyroid malignant lymphoma with huge chronic thyroiditis}

Takeshi Kusunoki, ${ }^{1}$ Hirotomo Homma, ${ }^{2}$

Yoshinobu Kidokoro, ${ }^{1}$

Akihisa Yoshikawa, ${ }^{1}$ Kumiko Tanaka, ${ }^{1}$

Satoko Kubo, ${ }^{1}$ Ryo Wada, ${ }^{2}$

Katsuhisa Ikeda ${ }^{3}$

${ }^{1}$ Department of Otorhinolaryngology, Juntendo University of Medicine, Shizuoka Hospital, Shizuoka;

${ }^{2}$ Department of Pathology, Juntendo University of Medicine, Shizuoka

Hospital, Shizuoka; ${ }^{3}$ Department of

Otorhinolaryngology, Juntendo

University of Medicine, Faculty of

Medicine, Tokyo, Japan

\begin{abstract}
We experienced a case of huge chronic thyroiditis with malignant lymphoma that caused dyspnea with tracheal stenosis, dysphagia with esophagus stenosis and recurrent nerve paralysis. In this case, thyroidectomy was performed and, after the surgery, there was no sign of breathing or swallowing difficulties, and it was confirmed by the postoperative computed tomography that the tracheal stenosis had improved. We considered two possible explanations for the preoperative right recurrent nerve paralysis. In the first, the right recurrent nerve could have suffered from mechanical stimulation such as compression and traction to the recurrent nerve due to enlargement of the malignant lymphoma together with chronic thyroiditis. The second possible explanation was that malignant cells had invaded neurons. We could not distinguish between the two possibilities, since this right recurrent nerve was spared and could not be examined histopathologically.
\end{abstract}

\section{Introduction}

Thyroid malignant tumors often cause difficulty in breathing because of the resulting tracheal stenosis and recurrent nerve paralysis. It has been reported that cases of thyroid malignant lymphoma coexisting with chronic thyroiditis could lead to rapid growth and result in difficulty in breathing due to tracheal stenosis. ${ }^{1,2}$ However, recurrent nerve paralysis with thyroid malignant lymphoma is quite rare. We experienced a case in which thyroid malignant lymphoma with a huge area of chronic thyroiditis caused respiratory and swallowing difficulties due to tracheal and esophagus stenosis, in addition to recurrent nerve paralysis. This case report presents the clinical findings, image examination, operative findings, histopathologic examination and other considerations.

\section{Case Report}

We present a case of an 84-year old, Japanese male patient, complaining for breathing difficulty and hoarseness.

\section{Preoperative course}

The patient had been administered thyroid hormone (levothyroxiline sodium $25 \mu \mathrm{g}$ /day) for chronic thyroiditis from three years before at the referring hospital. His goiter began increasing from one month before and his hoarseness, breathing and swallowing difficulties occurred within a month. This hospital introduced him to our hospital for examination and treatment. At the first examination, both of his thyroid lobes were enlarged (Figure 1, left). He complained of dyspnea in the neck anterior bending position. Fiberscopy showed right vocal cord paralysis but no tumor or inflamed lesions in the larynx or pharynx (Figure 1, middle). In the neck computed tomography (CT), both thyroid lobes showed diffuse swelling and the right lobe invaded into the anterior mediastinum. Moreover, this right lobe occupied the space between the posterior wall of the trachea and esophagus, creating pressure from the back of the trachea. Therefore, the circumference of his trachea was narrowed by the thyroid gland (Figure 1, right). CT, MRI and MRA did not show any apparent lesions in the neck, lung, mediastinum esophagus, aortic arch, brain or cerebrovasculature that could cause right recurrent nerve paralysis. Blood examination data were as shown in Table 1 . The thyroid function was hypothyroidism [freeT3: $2.5 \mathrm{pg} / \mathrm{mL}$ (normal range: 2.4-4.5pg/mL) free T4: $0.7 \mathrm{ng} / \mathrm{mL}$ (normal range: $1.0-1.7 \mathrm{ng} / \mathrm{mL}$ ), TSH: 32.0 (normal range: $0.56-4.30 \mu \mathrm{IU} / \mathrm{mL})]$. Thyroglobulin was $321 \mathrm{ng}$ (normal range: $0-33 \mathrm{ng} / \mathrm{mL}$ ). In the anti-thyroid autoantibodies, both the microsome test and thyroglobulin test were in the normal range (under 100). The diagnosis by fine needle aspiration cytology of the right lobe of the thyroid was chronic thyroiditis. However, soluble interleukin-2 receptor (sIL-2R) as a tumor marker of the lymphoma showed a high level (3319, normal range 122-496 U/mL). In the thyroid function examinations, hypothyroidism was
Correspondence: Takeshi Kusunoki, Department of Otorhinolaryngology, Juntendo University of Medicine, Shizuoka Hospital, 1129 Nagaoka Izunokuni-shi, Shizuoka 4102295, Japan. Fax: +81.55.948.5088.

E-mail: ttkusunoki001@aol.com

Key words: Tracheal stenosis; recurrent nerve paralysis; huge chronic thyroiditis; Hashimoto thyroiditis; dyspnea; dysphagia; thyroid lymphoma.

Acknowledgements: The authors thank $\mathrm{Mr}$ Brent Bell for reading the manuscript.

Contributions: TK, diagnosis and therapy, composition of this case report; HH, YK, AY, SK, therapy; RW, pathological diagnosis; KI, composition of this case report.

Conflicts of interests: The authors have no conflicts of interests to disclose.

Consent for publication: We confirm the patient's anonymity and obtained consent for publication of the clinical findings, image examination and pathological examination. A copy of this document is available for review by the editor of this journal.

Received for publication: 27 June 2020

Revision received: 18 September 2020.

Accepted for publication: 6 October 2020.

This work is licensed under a Creative Commons Attribution NonCommercial 4.0 License (CC BY-NC 4.0).

(C) Copyright: the Author(s), 2020

Licensee PAGEPress, Italy

Clinics and Practice 2020; 10:1276

doi:10.4081/cp.2020.1276

observed. Therefore, levothyroxiline sodium hydrate was increased from $25 \mu \mathrm{g} /$ day to $50 \mu \mathrm{g} /$ day and was able to correct the thyroid function to within the normal range.

We considered three potential causes of the right vocal cord paralysis as follows: i) A merger of idiopathic recurrent nerve paralysis; ii) The inflammation from the chronic thyroiditis might have spread, causing the recurrent nerve paralysis; iii) As a tumor marker of malignant lymphoma, the sIL-2R was at a high level. Therefore, the right recurrent nerve would be mechanically compressed by the rapidly increasing malignant thyroid lymphoma coexisting with chronic thyroiditis, resulting in paralysis. The patient received gradually deceased steroid therapy (prednisoline $30 \mathrm{mg} /$ day $\times 3$ day $\rightarrow 20 \mathrm{mg} /$ day $\times 3$ day $\rightarrow 10 \mathrm{mg} /$ day $\times 3$ day) in order to improve the tracheal stenosis, compression and edema of the recurrent nerve by reducing the enlargement of the thyroid lesions. However, the right recurrent nerve did not improve. 


\section{Operative findings}

We performed a right-thyroid lobectomy including the isthmus in order to improve the tracheal stenosis and for histopathologic examination. The right recurrent nerve with preoperative paralysis showed edema and was spared in the operation. The weight of the resected samples was $270 \mathrm{~g}$ (Figure 2, left). Intraoperative rapid pathology test suspected thyroid lymphoma.

\section{Postoperative course}

Postoperatively, the patient recovered from the breathing and swallowing difficulties, but the right recurrent nerve paralysis at pre-operation had not improved 4 months after surgery. The trachea and esophagus stenosis improved (Figure 2, middle). The postoperative histopathologic diagnosis was diffuse large B-cell lymphoma with chronic thyroiditis as the background (Figure 2, right). For the postoperative therapy, chemotherapy was planned by the department of hematology.

\section{Discussion}

It has been reported that some cases of chronic thyroiditis with dyspnea were associated with malignant thyroid lymphoma, which can cause tracheal stenosis due to its rapid increase. ${ }^{2,3}$ Most malignant thyroid lymphomas arise in chronic thyroiditis, as in our case. ${ }^{4,5}$ Our patient was 84 years-old. Taura ${ }^{1}$ insisted that, in middle age or older patients with increasing chronic thyroiditis, the possibility of malignant lymphoma should be considered. In general, the first choice for the treatment of chronic thyroiditis is administering a thyroid hormone agent. The indications of surgical treatment for chronic thyroiditis are the need for a biopsy or to release tracheal compression. The sIL-2R, as a tumor marker of malignant lymphoma, was at a high level and the chronic thyroiditis rapidly increased. Therefore, in our case, we suspected the possibility of coexisting malignant lymphoma in the chronic thyroiditis. The diagnosis by fine needle aspiration cytology for the right lobe of the thyroid was chronic thyroiditis. However, a diagnosis of malignant lymphoma requires histopathologic examination, including genetic and immunohistological study. Therefore, in order to release the stenosis of the tracheal and esophagus caused by the goiter and for the histopathologic diagnosis, we performed a right-thyroid lobectomy including the isthmus. The postoperative histopatho- logic diagnosis was diffuse large B-cell lymphoma with chronic thyroiditis as the background. In our case, thyroid lesions invaded the space between the posterior wall of the trachea and esophagus causing pressure from the back of the trachea. This condition was considered to be the cause of the breathing and swallowing difficulties.
A case of recurrent nerve paralysis with malignant thyroid lymphoma was previously reported by Jiu. ${ }^{6}$ His patient had complete recovery of vocal cord function following radiation therapy for a large thyroid lymphoma associated with vocal cord paralysis. He described that the rather rapid and complete recovery of the vocal cord func-

Table 1. Laboratory results.

\begin{tabular}{lll}
\hline Free T3 & $2.5 \mathrm{pg} / \mathrm{mL}$ & (normal range: $2.4-4.5 \mathrm{pg} / \mathrm{mL}$ ) \\
Free T4 & $0.7 \mathrm{ng} / \mathrm{mL}$ & (normal range: $1.0-1.7 \mathrm{ng} / \mathrm{mL}$ ) \\
\hline TSH & $32.0 \mathrm{IU} / \mathrm{mL}$ & (normal range: $0.56-4.30 \mathrm{IU} / \mathrm{mL}$ ) \\
Thyroglobulin & $321 \mathrm{ng} / \mathrm{mL}$ & (normal range: $0-33 \mathrm{ng} / \mathrm{mL}$ ) \\
\hline Microsome test & Under 100 & (normal range: under 100 ) \\
Thyroglobulin test & Under 100 & (normal range: under 100 ) \\
\hline sIL-2R & $3319 \mathrm{U} / \mathrm{mL}$ & (normal range $122-496 \mathrm{U} / \mathrm{mL}$ ) \\
\hline
\end{tabular}
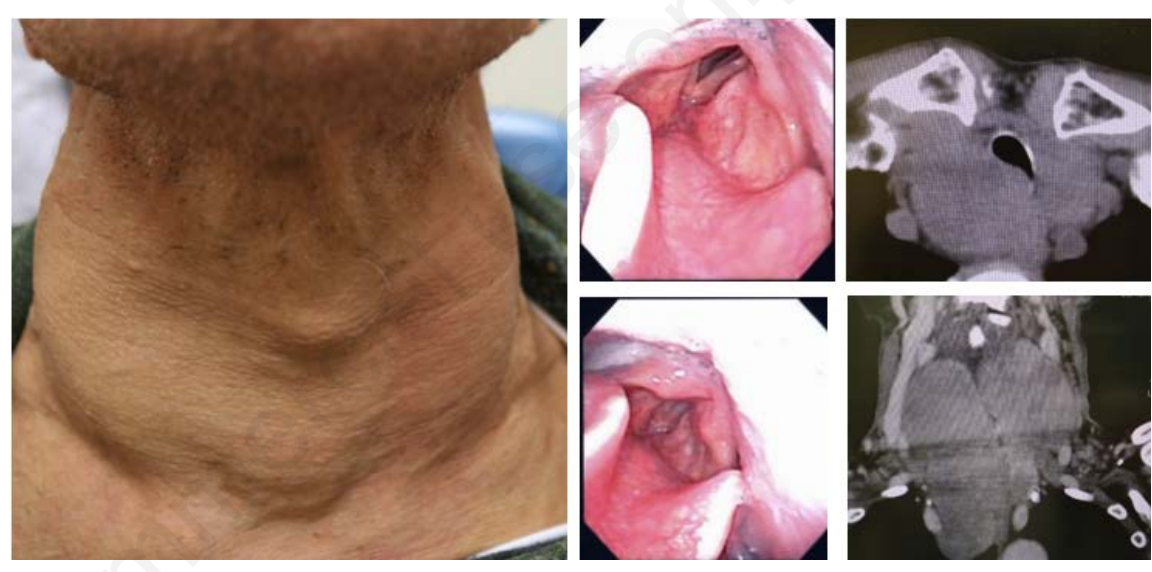

Figure 1. Both thyroid lobes showed diffuse swelling (left). Fiberscopy showed right vocal cord paralysis but no tumor and inflamed lesions in the larynx and pharynx (middle). Computed tomography showed that bilateral thyroid lobes were much enlarged and occupied the space between the posterior wall of the trachea and esophagus with pressure from the back of the trachea (right).
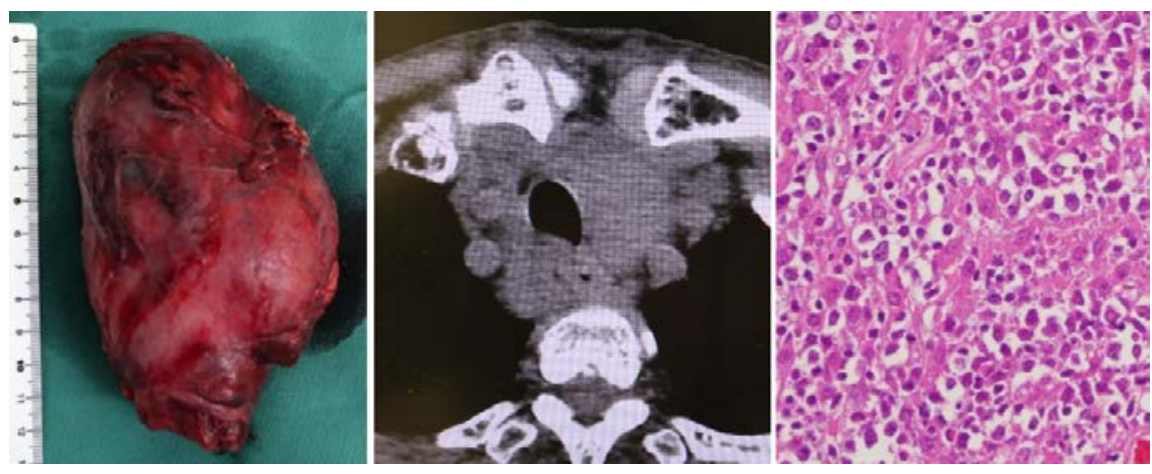

Figure 2. Resected sample (left). Histological examination (HE staining) of the resected sample showed diffuse large B-cell lymphoma with chronic thyroiditis as the background. Atypical circular cell nests were found in the lesions of malignant thyroid lymphoma (middle). Postoperative computed tomography showed improvement of the trachea stenosis (right). 
tion suggested that the thyroid lymphoma would cause paralysis merely by reversible compression, rather than by neural invasion or tumor-induced neurolysis. He performed only a core needle biopsy of the thyroid, but not thyroidectomy. In our operative findings, the right recurrent nerve with preoperative paralysis showed edema but was spared. This right recurrent nerve paralysis had not improved 4 months after surgery. We also considered two possible causes of the preoperative right recurrent nerve paralysis. One possibility was that the right recurrent nerve suffered from mechanical stimulation such as compression and traction due to the enlargement of the malignant lymphoma together with the chronic thyroiditis. The second possibility was that malignant cells invaded the neurons. We could not distinguish between the two since the right recurrent nerve was spared in our operation and could not be examined histopathologically.

\section{References}

1. Taura M, Kamizono K, Ejma M, et al. Primary thyroid lymphoma: A report of three cases. Otologia Fukuoka 2010;56:243-6.

2. Nomizu T, Matsuzaki M, Katagata N, et al. A case of malignant lymphoma of the neck which presented the huge struma. JJAES JSTS 2014;31:134-8.
3. Thieblemont C, Mayer A, Dumontet C, et al. Primary thyroid lymphoma is a heterogeneous disease. $\mathrm{J}$ Clin Endocrinol Metab 2002;87:105-11.

4. Matsuzuka F, Miyauchi A, Katayama S, et al. Clinical aspects of primary thyroid lymphoma: Diagnosis and treatment based on our experience of 119 cases. Thyroid 1993;3;93-9.

5. Aozasa K. Malignant lymphomas of the thyroid glands- Analysis of 79 patients with emphyasis on histologic prognostic factors. Cancer 1986;58:100-4.

6. Jiu JB, Soboi SM, Grozea PN. Vocal cord paralysis and recovery with thyroid lymphoma. Laryngoscope 1985 ; 95:57-9. 